\title{
Bisociative Music Discovery and Recommendation
}

\author{
Sebastian Stober, Stefan Haun, and Andreas Nürnberger \\ Data \& Knowledge Engineering Group, Faculty of Computer Science, \\ Otto-von-Guericke-University Magdeburg, D-39106 Magdeburg, Germany \\ \{sebastian.stober, stefan.haun, andreas. nuernberger\} @ovgu.de
}

\begin{abstract}
Surprising a user with unexpected and fortunate recommendations is a key challenge for recommender systems. Motivated by the concept of bisociations, we propose ways to create an environment where such serendipitous recommendations become more likely. As application domain we focus on music recommendation using MusicGalaxy, an adaptive user-interface for exploring music collections. It leverages a nonlinear multi-focus distortion technique that adaptively highlights related music tracks in a projection-based collection visualization depending on the current region of interest. While originally developed to alleviate the impact of inevitable projection errors, it can also adapt according to user-preferences. We discuss how using this technique beyond its original purpose can create distortions of the visualization that facilitate bisociative music discovery.
\end{abstract}

\section{Introduction}

One of the big challenges of computer science in the 21st century is the digital media explosion. Online music stores already contain several millions of music tracks and steadily growing hard-drives are filled with personal music collections of which a large portion is almost never used. Music recommender systems aim to help us cope with this amount of data and find new interesting music or rediscover once loved pieces we have forgotten about - a task also called "recomindation" 22]. One common problem that many recommender systems face is that their recommendations are often too obvious and thus not particularly useful when it comes to discovering new music. Especially, collaborative filtering approaches are prone to a strong popularity bias [2]. In fact, McNee et al. argue that there is too much focus on improving the accuracy of recommender systems. They identify several important aspects of human-recommender interaction of which serendipity is specifically related to the above phenomenon [17. A serendipitous recommendation is unexpected and fortunate - something that is particularly hard to grasp and evaluate.

We recently conducted a user study to assess the usability and usefulness of a visualization technique for the exploration of large multimedia collections. One task was to find photographs of lizards in a collection of photos taken in Western Australia. The user-interface was supposed to support the participants 


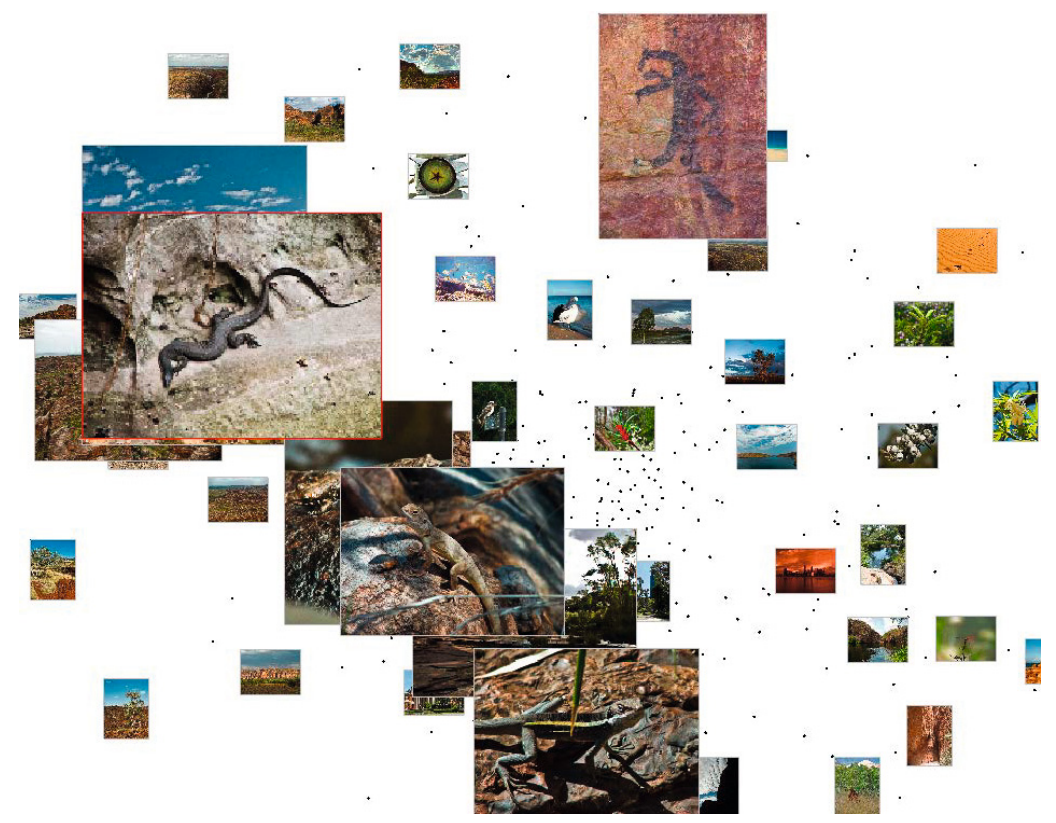

Fig. 1. Serendipitous encounter with a rock painting of a lizard when looking for photographs of a lizard (using the Adaptive SpringLens visualization for exploring multimedia collections [26])

by pointing out possibly relevant photos for a seed photo. As it happened, one of the participants encountered a funny incident: While looking for photographs showing a lizard, he selected an image of a monitor lizard as seed. To his surprise, the system retrieved an image showing the rock painting of a lizard (Figure 1). Interestingly, rock paintings were actually another topic to find photos for and the relevant photos were a lot harder to make out in the collection than the lizards. Bearing in mind that according to Isaac Asimov "the most exciting phrase to hear in science, the one that heralds new discoveries, is not 'Eureka!' (I found it!) but 'That's funny ...' ", we decided to further investigate this phenomenon. What the participant encountered is called a bisociation - a bridging element between the two distinct domains: animals and rock paintings. While most associations are found between concepts of one domain, there are certain paths which either bridge two different domains or connect concepts by incorporating another domain. In his book The Act of Creation, Arthur Köstler, an Austrian publisher, coined the term bisociation for these types of associations and as it turns out, many scientific discoveries are in some way bisociations 9 .

Admittedly, no one expects scientific discoveries from a music recommender application. However, the question persists whether we can leverage the effect of bisociations and create an environment where serendipitous recommendations become more likely. After all, the concept of bisociation is much easier to grasp than serendipity and can even be formalized by means of graph theory [10]. 
This paper is structured as follows: Section 2 points out related work in the field of exploratory music discovery and recommendation. Section 3 briefly reviews the MusicGalaxy user-interface based on the Adaptive SpringLens visualization technique that we have developed in previous work and evaluated in the above mentioned user study. Based on this foundation, Section 4 describes how the MusicGalaxy user-interface can be turned into an environment that supports bisociative music discovery. Finally, Section 5 discusses early findings and Section 6] concludes the paper.

\section{Related Work}

There is a variety of approaches to music discovery and recommendation that rely on some way of collection exploration. Generally, there are several possible aspects - each with different levels of abstraction - that can be supported, the most common being: track, album, artist and genre. Though a system may cover more than one aspect (e.g., in 31] visualized as disc or TreeMap), usually a single one is chosen. In this paper, the focus is on the track level but knowledge about relations to artists and albums is also incorporated.

\subsection{Interfaces for Creative Music Discovery}

MusicRainbow [19] is an interface to explore music collections at the artist level. Using a traveling salesman algorithm, similar artists are mapped near each other on a circular rainbow where the colors of the rainbow reflect the genres. Audiobased similarity is combined with words extracted from web pages related to the artists. The words are used to label the rainbow and describe the artists.

MusicSun 20] applies a similar concept to discover artists. Recommendations are based on one or more artists that are selected by the user and displayed in the center of a sun. The sun rays (triangles) represent words that describe these seed artists. The size of a ray's base reflects how well the respective word fits to the artist and its length is proportional to the number of artists in the collection that can also be described by that word. Selecting a ray, a list of recommended artists is generated. Similarly to the work presented in this paper, users can also adapt the impact of three different aspects of music similarity that are combined.

Musicream [7] facilitates active, flexible, and unexpected encounters with musical pieces by extending the common concept of query by example: Several tubs provide streams of music pieces (visualized as discs) that the user can grab and drop into the playback region of the interface or use as a magnet to filter similar pieces from the streams. The interface also provides enhanced playback functions such as building playlists of playlists or going back to any previous point in the play history.

The MusicExplorer FX 11 takes a different approach: Built upon the EchoNest AP12, it displays a local similarity graph, connecting an artist with the most

\footnotetext{
1 http://musicexplorerfx.citytechinc.com/

2 http://developer.echonest.com/
} 
similar ones. The interface also shows a navigation history containing the previously visited artists. A similar approach is taken by the Relational Artist Map RAMA 24 that additionally displays labels as graph overlay. However, both lack a global overview of the whole artist space and users need to specify a seed artist to start with. In contrast to this, the Last.fm artist map ${ }^{3}$ displays the whole graph (based on the Last.fm AP4). As this results in a lot of clutter caused by crossing edges, it is hard to navigate and explore the graph. Consequently, it is rather suited to map a user's listening preferences.

\subsection{Projection of a Similarity Space}

In contrast to the already described works, the visualization approach taken here is primarily based on a projection of a similarity space. This is a very common method to create an overview of a collection. Popular dimensionality reduction techniques applied are self-organizing maps (SOM) 218 18 15 27] principal component analysis (PCA) [13] and multidimensional scaling (MDS) or similar force-based approaches 12414 . Mapping the collection from high-dimensional feature/similarity space onto display space, it is usually impossibly to correctly preserve all distances (independent of the method used). Some objects will appear closer than they actually are and on the other side, some objects that are distant in the projection may in fact be neighbors in the original space 5 Only a small number of approaches tries to additionally visualize such properties of the projection itself: The MusicMiner [18] draws mountain ranges between songs that are displayed close to each other but are dissimilar. The SoniXplorer [15] uses the same geographical metaphor but in a 3D virtual environment that the user can navigate with a game pad. The "Islands of Music" 21] and its related approaches 84] use the third dimension the other way around: Here, islands or mountains refer to regions of similar songs (with high density). Both ways, local properties of the projection are visualized - neighborhoods of either dissimilar or similar songs. Soundbite [14, on the other hand, attempts to visualize properties of the projection that are not locally confined: For selected objects in the (MDS) projection, edges are drawn additionally that connect them to their nearest neighbors - according to the underlying similarity and not the distance in the projection. We take a similar approach, interpreting connections between neighbors that are distant in the projection as "wormholes" through the high-dimensional feature space in analogy to the concept in astrophysics.

\subsection{User-Adaption during the Exploration Process}

Additionally, our goal is to support user-adaptation during the exploration process by means of weighting aspects of music similarity. Of the above approaches,

\footnotetext{
3 http://sixdegrees.hu/last.fm/interactive_map.html

4 http://www.last.fm/api

${ }^{5}$ Note that it is impossible to fix these problems without causing damage elsewhere as the projection is in general already optimal with respect to the projection technique applied.
} 


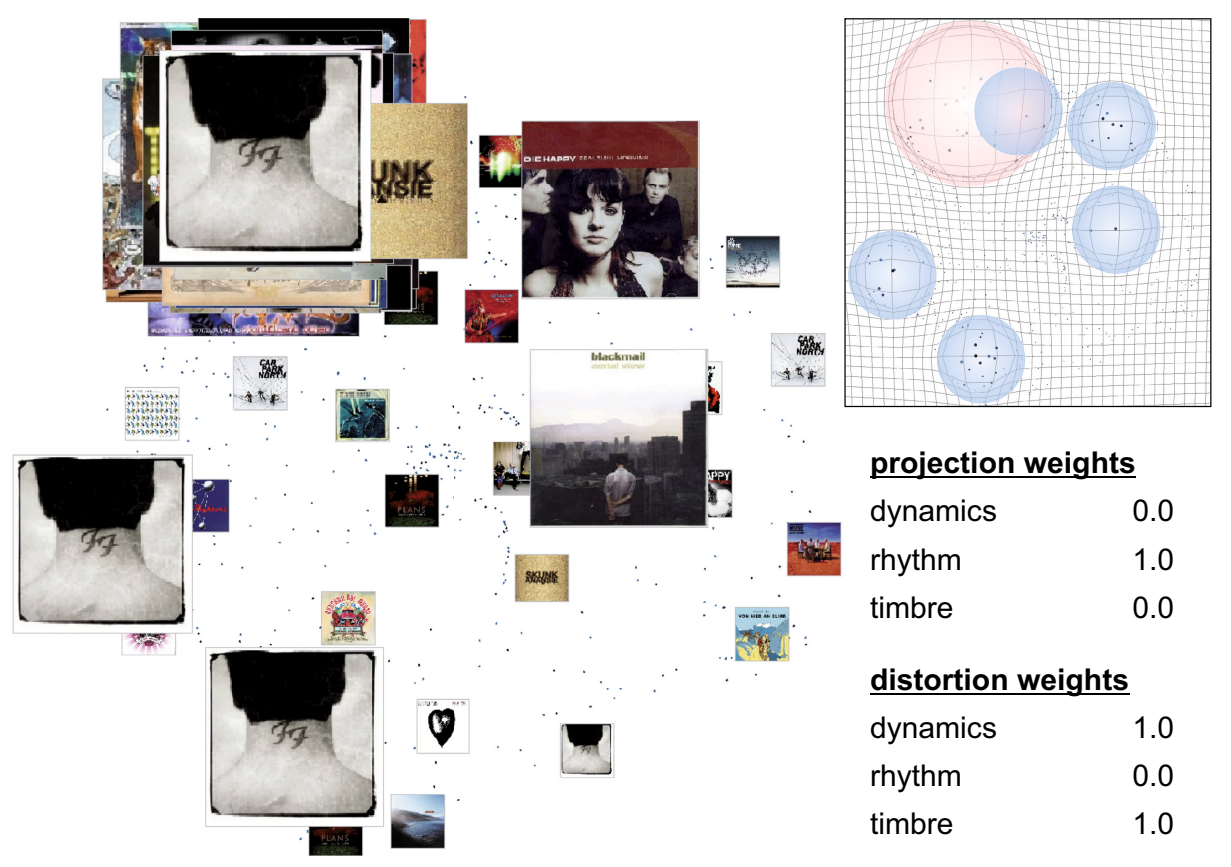

Fig. 2. Left: MusicGalaxy visualization. Top right: corresponding SpringLens distortion resulting from primary focus (red) and 5 secondary lenses (blue). Bottom right: facet weights for the projection and distortion distance measures.

only the revised SoniXplorer [15, MusicBox [13, MusicSun [20] and our original SOM-based prototype 27] allow automatic adaptation of the view on the collection through interaction. Apart from this, there exist systems that also adapt a similarity measure but not to change the way the collection is presented in an overview but to directly generate playlists (e.g., [132]). In contrast to these systems that purely focus on the task of playlist generation, we pursuit a more general goal in providing an adaptive overview of the collection that can then be used to easily generate playlists as, e.g., already shown in [8] or [13].

\section{The MusicGalaxy Visualization}

In previous work [28]29, we have developed an interface for exploring large music collections using a galaxy metaphor that addresses the problem of distorted neighborhoods. Figure 2 shows a screenshot of the interface visualizing a music collection 6 Each track is displayed as a star, i.e., a point, with its brightness and - to some extend - its hue depending on a predefined importance measure (here a play count obtained from last.fm - other measures such as a general popularity or ratings are possible). A spatially well distributed subset of the

$\overline{{ }^{6} \text { A demo video is available at http://www.dke-research.de/aucoma }}$ 
collection (specified by filters) is additionally displayed as an album cover for orientation. The arrangement of the stars is computed using multi-dimensional scaling (MDS) 11 relying on a set of descriptive features to be extracted beforehand 7 MDS is a popular neighborhood-preserving projection technique that attempts to preserve the distances (dissimilarities) between the objects in the projection. The result of the MDS is optimal with respect to the minimization of the overall distance distortions. Thus, fixing one distorted neighborhood is not possible without damaging others. However, if the user shows interest in a specific neighborhood, this one can get a higher priority and be temporarily fixed (to some extend) at the cost of the other neighborhoods. To this end, an adaptive distortion technique called SpringLens [5] is applied that is guided by the user's focus of interest. The SpringLens is a complex overlay of multiple fish-eye lenses divided into primary and secondary focus. The primary focus is a single large fish-eye lens used to zoom into regions of interest. At the same time, it compacts the surrounding space but does not hide it from the user to preserve overview. While the user can control the primary focus, the secondary focus is automatically adapted. It consists of a varying number of smaller fisheye lenses. When the primary focus changes, a neighbor index is queried with the object closest to the center of focus. If nearest neighbors are returned that are not in the primary focus, secondary lenses are added at the respective positions. As a result, the overall distortion of the visualization temporarily brings the distant nearest neighbors back closer to the focused region of interest. This way, distorted distances introduced by the projection can to some extend be compensated.

The user-interface has been evaluated in a study as reported in 26. In the study, 30 participants had to solve an exploratory image retrieval task 8 : Each participant was asked to find representative images for five non-overlapping topics in a collection containing 350 photographs. This was repeated on three different collections - each one with different topics and with varying possibilities for interaction, comparing the fish-eye with traditional panning and zooming and a combination thereof. In total, each participant spent between 30 and 60 minutes using the system. The participants clearly preferred the fish-eye and the combined interface over the traditional panning and zooming in terms of helpfulness, simplicity and intuitivity. Further, gaze information recorded with an eye-tracker showed extensive use of the secondary focus to find more relevant images belonging to the same topic as the one in primary focus. As anticipated, some participants used the primary lens to skim through the photo collection in a rather continuous fashion. But surprisingly, there was also a group that browsed the collection mostly by moving (in a single click) the primary focus to some (previously) secondary focus region step-by-step - much like navigating an invisible neighborhood graph. Thus, it can

\footnotetext{
$\overline{7}$ Alternatively, feature information may also be annotated manually or collected from external sources.

${ }^{8}$ Images were used instead of tracks because (1) it could be guaranteed that the collection is unknown to all users and (2) visual similarity and relevance are much quicker assessed.
} 
be concluded that the multi-focus SpringLens technique is very well suited for exploratory recommendation scenarios.

An aspect not addressed in the user study is that MusicGalaxy additionally allows to adapt the underlying music similarity. To this end, music similarity is represented as a distance measure that is a weighted linear combination of facet distances. Each facet covers a specific aspect of music similarity such as melody, harmony, rhythm, dynamics or lyrics and is defined by one ore more representative features and an appropriate distance measure. The importance of the individual facets can be adapted by changing their weights for the linear aggregation. To this end, the user interface has a control panel (not shown in the screenshot) with respective sliders. As we have shown in recent experiments with simulated user-interaction [30, it is also possible to adapt the weights automatically based on (relative) preferences derived from user actions such as judging two objects to be more similar with respect to a third one. Using adaptation, it becomes possible to personalize the music similarity measure used for recommendations.

\section{Bisociative Lens Distortions}

How can MusicGalaxy be turned into an environment that supports bisociative music discovery? The general idea is to combine two distinct domain views into one visualization by using the secondary focus to highlight connections to nearest neighbors in a different domain than the one used for projection: The "primary domain" is directly visualized by the projection and contains the displayed tracks connected by neighborhood relations that are implicitly induced between each track and its neighbors in the projection 9 Additionally the "secondary domain" - which is used to identify nearest neighbors for the secondary focus distortion - is not directly visible to the user. A bisociation occurs in this setting if two tracks are not neighbors in the projection domain, i.e., close to each other in the display, but are connected in the secondary domain. In this case, the secondary focus will highlight this connection by focusing on the bisociated track - or similar image with respect to another domain as shown in Figure 1.

\subsection{Orthogonal Similarity Measures}

The simplest way to create such a setting is to use orthogonal similarity measures, i.e., defined on non-overlapping facet sets, for the two domains by choosing the facet weights accordingly. E.g., in Figure 2 the tracks in secondary focus are very different in rhythm (large distance in projection) but very similar in dynamics and timbre with respect to the track in primary focus. This approach could also be used in different applications. To illustrate the possibilities, imagine a user wants to explore a collection of world-music as, e.g., addressed by

${ }^{9}$ This is rather an artificial mental model a user perceives as no connections are explicitly visualized. Due to possible distortions introduced by dimensionality reduction, it only approximates the one derived from the actual distances in the original space. 
mHashup [16]. In such applications, a straightforward way for the arrangement of the tracks would be according to their geographical origin, i.e., mapping the tracks on a common world map. Using this primary domain instantly gives the user an overview of the geographic distribution of the tracks in the collection. With the primary fish-eye lens, the user could magnify a region he is interested in. This would allow to display the local distribution of tracks in more detail and differentiate smaller (sub)regions. Note that in this special case, the arrangement of the tracks is perfect in the sense that all distances can be displayed distortionfree (except for the neglectible mapping of the earth's surface to a plane) because there is no dimensionality reduction involved. The secondary focus in its original setting would be unnecessary here anyway and it could therefore be freely used to highlight regions with nearest neighbors with respect to other aspects addressed by the secondary domain - e.g., acoustic similarity as a combination of its respective facets. Further, analyzing the interaction with the user, the system can - over time - learn which (acoustic) facets (of the secondary domain) are particularly important for the user and personalize the similarity measure for nearest neighbor retrieval accordingly. This has already been described and evaluated in 30 .

\subsection{Generalization to Domain Graphs}

The above example uses an orthogonal similarity measure for the secondary domain. This is, however, only a very special case. Generally, the secondary domain might be any graph that contains at least the tracks as concepts (nodes) and allows to find neighboring tracks by some way of traversing relations between the concepts. An orthogonal similarity measure as described above induces such a graph: In this case, the graph contains only the tracks as concepts plus relations between tracks that are nearest neighbors and finding nearest neighbors for a track means simply returning all directly related tracks. An alternative way to construct such a sparse neighborhood graph for the secondary domain is to use any (black-box) system that recommends similar tracks for a seed track or even a combination of several such systems. However, the graph does not need to be confined to tracks. In fact, it may be arbitrarily complex - e.g., contain also artists, albums plus respective relations and possibly allowing multiple paths between tracks. For instance, from the freely available data from MusicBrainz10, a user maintained community music meta-data base, a large graph can be constructed containing more than $10 \mathrm{M}$ tracks, $740 \mathrm{~K}$ albums, $600 \mathrm{~K}$ artists and $48 \mathrm{~K}$ labels 11 Between these entities, common relationships exist that, e.g., link tracks to artists and albums as well as albums to artists and labels. Apart from this, a large variety of advanced relationships links (ARL) exists. They are particularly interesting as they go beyond trivial information, such as links from tracks and albums to mastering and recording engineers, producers and studios (in total more than $281 \mathrm{~K}$ artist-album and $786 \mathrm{~K}$ artist-recording ARLs), how artists are

\footnotetext{
10 http://musicbrainz.org/

11 Figures as of January 2011 when the graph was created.
} 
related with each other (more than 135K ARLs), or which tracks contain samples of others (more than $44 \mathrm{~K}$ recording-recording ARLs) 12

Nearest neighbors for a track in primary focus can be found by traversing the MusicBrainz graph in breadth-first order collecting paths to other tracks. Graph traversal stops when either the traversal depth or the number of reached track nodes exceeds a predefined threshold. As only the most relevant tracks can be highlighted by the secondary focus, some relevance measure is required to rank the retrieved tracks. Because increasing serendipity is the main objective, the relevance measure should capture how likely a track will be a lucky surprise for the user. This is however all but trivial. Possible simple heuristics are:

- Prefer tracks that are projected far away from the primary focus (and thus most likely sound very different).

- Prefer tracks that the user has not listened to a lot or for a long time (and probably is no longer aware of).

- Prefer tracks of different artists and/or albums.

The result of using either heuristic or a combination thereof will most likely surprise the user but at the same time the risk is high that the connection to the primary focus is too far fetched. Therefore, paths need to be judged according to their interestingness. Platt [23] defines discrete edge distances depending on the type of relationships for a similar graph created on a dataset from the All Music Guide 3]. Similar weightings can be applied here. Alternatively, weights could be assigned to common path patterns instead - possibly penalizing longer paths. For instance, some path patterns are straightforward such as track-artisttrack (same artist) or track-album-track (same album) where the latter is more interesting in terms of serendipity because it could be a compilation that also contains tracks of other artists. Both weighting approaches require empirical tuning of the respective weights. Another option is to count the frequencies of occurring path patterns and boost infrequent and thus remarkable patters which can be interpreted as analogy to the idf weights used in text retrieval. This favors patterns containing ARLs. If multiple paths between two tracks are found, their weights can be aggregated, e.g., using the maximum, minimum or average. More sophisticated methods like those described in [25] are currently developed to facilitate bisociations on text collections and could also be applied here to further increase the chances of bisociative recommendations from complex domain graphs. This is currently studied more thoroughly as the impact of the different heuristics and the values of their respective parameters are not yet fully clear.

\section{Discussion}

This research in the field of bisociative music collection exploration is still in an early stage and clearly leaves several options for elaboration. For instance, it would be possible to extend the domain graph beyond MusicBrainz by incorporating information from other sources such as last.fm, The EchoNest or Myspace

${ }^{12}$ Full list available at: http://wiki.musicbrainz.org/Category:Relationship_Type 
(see Section 2 for some graphs created from artist-similarity relations that can be obtained from these resources).

The user-interface needs to better integrate the graph information - possibly displaying (single) interesting connections. It can also be important to point out why a specific track is highlighted by the secondary focus. Such explanations would make the recommendation more understandable and less ambiguous. Currently, a user can only recognize tracks of the same album (because of the same cover) and to some extend tracks of the same artists (given he can associate the album covers with the respective artists). Looking at the screenshot of $\mathrm{Mu}-$ sicGalaxy shown in Figure 2, four tracks from the same album can be seen in secondary focus. This is in fact because of a strong album effect (the album contains jazz cover versions of Beatles songs) captured entirely only by acoustic facets and without knowledge of track-album or track-artist relations. However, a similar result could have been produced by using the MusicBrainz graph as secondary domain. There is currently no visual clue to differentiate one from the other. A deeper analysis of the relationship graph could lead to more sophisticated ways of judging the interestingness of paths to related tracks. In order to personalize recommendations and increase the chance of surprises, additional information from a user-profile could be incorporated. Finally, it is necessary to test the proposed approach in another user study. However, it still remains an open question how to objectively judge the quality of recommendations in terms of serendipity 13

\section{Conclusions}

This paper described an approach to increase the chance of serendipitous recommendations in an exploratory music retrieval scenario. Instead of addressing serendipity directly, we proposed to exploit the related concept of bisociations that can be formalized by means of graph theory. We demonstrated how separating the underlying similarity measures for projection and distortion in the MusicGalaxy interface makes is possible to link two distinct domain views on a music collection - creating a setting that promotes bisociations where serendipitous recommendations become more likely. We hope that this paper can contribute to the ongoing discussion of improving the serendipity of recommendations and at the same time spreads the awareness of the bisociation concept.

Acknowledgments. The authors would like to thank the members of the EU BISON project for many fruitful discussions on the topic of bisociation. This work was supported in part by the German National Merit Foundation, the German Research Foundation (DFG) project AUCOMA and the European Commission under FP7-ICT-2007-C FET-Open, contract no. BISON-211898.

Open Access. This article is distributed under the terms of the Creative Commons Attribution Noncommercial License which permits any noncommercial use, distribution, and reproduction in any medium, provided the original author(s) and source are credited.

${ }^{13}$ For a general discussion about the evaluation of exploratory user interfaces see 6 . 


\section{References}

1. Baumann, S., Halloran, J.: An ecological approach to multimodal subjective music similarity perception. In: Proc. of 1st Conf. on Interdisc. Musicology, CIM 2004 (2004)

2. Celma, O., Cano, P.: From hits to niches?: or how popular artists can bias music recommendation and discovery. In: Proc. of the 2nd KDD Workshop on LargeScale Recommender Systems and the Netflix Prize Competition, NETFLIX 2008 (2008)

3. Ellis, D.P.W., Whitman, B., Berenzweig, A., Lawrence, S.: The quest for ground truth in musical artist similarity. In: Proc. of 3rd Int. Conf. on Music Information Retrieval, ISMIR 2002 (2002)

4. Gasser, M., Flexer, A.: Fm4 soundpark audio-based music recommendation in everyday use. In: Proc. of the 6th Sound and Music Computing Conference, SMC 2009 (2009)

5. Germer, T., Götzelmann, T., Spindler, M., Strothotte, T.: Springlens: Distributed nonlinear magnifications. In: Eurographics 2006 - Short Papers, pp. 123-126. Eurographics Association, Aire-la-Ville, Switzerland (2006)

6. Gossen, T., Nitsche, M., Haun, S., Nürnberger, A.: Data exploration for bisociative knowledge discovery: A brief overview of tools and evaluation methods. In: Berthold, M.R. (ed.) Bisociative Knowledge Discovery. LNCS (LNAI), vol. 7250, pp. 287-300. Springer, Heidelberg (2012)

7. Goto, M., Goto, T.: Musicream: New music playback interface for streaming, sticking, sorting, and recalling musical pieces. In: Proc. of 6th Int. Conf. on Music Information Retrieval, ISMIR 2005 (2005)

8. Knees, P., Pohle, T., Schedl, M., Widmer, G.: Exploring Music Collections in Virtual Landscapes. IEEE MultiMedia 14(3), 46-54 (2007)

9. Köstler, A.: The Act of Creation. Macmillan (1964)

10. Kötter, T., Thiel, K., Berthold, M.: Domain bridging associations support creativity. In: Proc. 1st Int. Conf. on Computational Creativity (ICCC 2010), Lisbon (2010)

11. Kruskal, J., Wish, M.: Multidimensional scaling. Sage (1986)

12. Lamere, P., Eck, D.: Using 3D visualizations to explore and discover music. In: Proc. of 8th Int. Conf. on Music Information Retrieval (ISMIR 2007), pp. 173-174 (2007)

13. Lillie, A.S.: Musicbox: Navigating the space of your music. Master's thesis, MIT (2008)

14. Lloyd, S.: Automatic playlist generation and music library visualisation with timbral similarity measures. Master's thesis, Queen Mary University of London (2009)

15. Lübbers, D., Jarke, M.: Adaptive multimodal exploration of music collections. In: Proc. of 10th Int. Conf. on Music Information Retrieval, ISMIR 2009 (2009)

16. Magas, M., Casey, M., Rhodes, C.: mhashup: fast visual music discovery via locality sensitive hashing. In: SIGGRAPH 2008: ACM SIGGRAPH 2008 New Tech Demos, p. 1. ACM, New York (2008)

17. McNee, S.M., Riedl, J., Konstan, J.A.: Making recommendations better: an analytic model for human-recommender interaction. In: CHI 2006 Extended Abstracts on Human Factors in Computing Systems (2006)

18. Mörchen, F., Ultsch, A., Nöcker, M., Stamm, C.: Databionic visualization of music collections according to perceptual distance. In: Proc. of 6th Int. Conf. on Music Information Retrieval, ISMIR 2005 (2005) 
19. Pampalk, E., Goto, M.: Musicrainbow: A new user interface to discover artists using audio-based similarity and web-based labeling. In: Proc. of 7th Int. Conf. on Music Information Retrieval (ISMIR 2006), pp. 367-370 (2006)

20. Pampalk, E., Goto, M.: Musicsun: A new approach to artist recommendation. In: Proc. of 8th Int. Conf. on Music Information Retrieval (ISMIR 2007), pp. 101-104 (2007)

21. Pampalk, E., Rauber, A., Merkl, D.: Content-based organization and visualization of music archives. In: Proc. of ACM MULTIMEDIA 2002 (2002)

22. Plate, C., Basselin, N., Kröner, A., Schneider, M., Baldes, S., Dimitrova, V., Jameson, A.: Recomindation: New Functions for Augmented Memories. In: Wade, V.P., Ashman, H., Smyth, B. (eds.) AH 2006. LNCS, vol. 4018, pp. 141-150. Springer, Heidelberg (2006)

23. Platt, J.C.: Fast embedding of sparse music similarity graphs. In: Advances in Neural Information Processing Systems (NIPS 2003), vol. 16 (2004)

24. Sarmento, L., Gouyon, F., Costa, B., Oliveira, E.: Visualizing networks of music artists with rama. In: Int. Conf. on Web Information Systems and Technologies, Lisbon (2009)

25. Segond, M., Borgelt, C.: Selecting the links in bisoNets generated from document collections. In: Berthold, M.R. (ed.) Bisociative Knowledge Discovery. LNCS (LNAI), vol. 7250, pp. 56-67. Springer, Heidelberg (2012)

26. Stober, S., Hentschel, C., Nürnberger, A.: Evaluation of adaptive springlens - a multi-focus interface for exploring multimedia collections. In: Proc. of 6th Nordic Conf. on Human-Computer Interaction, NordiCHI 2010 (2010)

27. Stober, S., Nürnberger, A.: Towards User-Adaptive Structuring and Organization of Music Collections. In: Detyniecki, M., Leiner, U., Nürnberger, A. (eds.) AMR 2008. LNCS, vol. 5811, pp. 53-65. Springer, Heidelberg (2010)

28. Stober, S., Nürnberger, A.: A multi-focus zoomable interface for multi-facet exploration of music collections. In: Proc. of 7 th Int. Symposium on Computer Music Modeling and Retrieval, CMMR 2010 (2010)

29. Stober, S., Nürnberger, A.: MusicGalaxy - an adaptive user-interface for exploratory music retrieval. In: Proc. of 7th Sound and Music Computing Conference, SMC 2010 (2010)

30. Stober, S., Nürnberger, A.: Similarity Adaptation in an Exploratory Retrieval Scenario. In: Detyniecki, M., Knees, P., Nürnberger, A., Schedl, M., Stober, S. (eds.) AMR 2010. LNCS, vol. 6817, pp. 144-158. Springer, Heidelberg (2012)

31. Torrens, M., Hertzog, P., Arcos, J.L.: Visualizing and exploring personal music libraries. In: Proc. of 5th Int. Conf. on Music Information Retrieval, ISMIR 2004 (2004)

32. Vignoli, F., Pauws, S.: A music retrieval system based on user driven similarity and its evaluation. In: Proc. of 6th Int. Conf. on Music Information Retrieval, ISMIR 2005 (2005) 